Research Article

\title{
Global Dynamics of a Generalized SIRS Epidemic Model with Constant Immigration
}

\author{
Qianqian Cui $\mathbb{D}$, Qinghui Du, and Li Wang $(\mathbb{D}$ \\ School of Mathematics and Statistics, Ningxia University, Yinchuan 750021, China \\ Correspondence should be addressed to Li Wang; wangli229@126.com
}

Received 31 May 2020; Revised 13 October 2020; Accepted 20 October 2020; Published 23 November 2020

Academic Editor: Fotios Georgiades

Copyright ( 92020 Qianqian Cui et al. This is an open access article distributed under the Creative Commons Attribution License, which permits unrestricted use, distribution, and reproduction in any medium, provided the original work is properly cited.

In this paper, we discuss the global dynamics of a general susceptible-infected-recovered-susceptible (SIRS) epidemic model. By using LaSalle's invariance principle and Lyapunov direct method, the global stability of equilibria is completely established. If there is no input of infectious individuals, the dynamical behaviors completely depend on the basic reproduction number. If there exists input of infectious individuals, the unique equilibrium of model is endemic equilibrium and is globally asymptotically stable. Once one place has imported a disease case, then it may become outbreak after that. Numerical simulations are presented to expound and complement our theoretical conclusions.

\section{Introduction}

In 1927, Kermack and McKendrick [1] proposed the compartment dynamic model, which uses differential equations research the dissemination rule of the infectious diseases. Since then, infectious disease dynamics has been developing vigorously and produced some important theoretical and practical valuable results. In classical KermackMckendrick model, the whole population is divided into three subclasses: susceptible, infected, and recovered, which is also called SIR-type model. This type of model always assumed that the recovered individuals have permanent immunity. In fact, a plenty of diseases just have only a short period of acquired immunity, such as flu, tuberculosis, Hepatitis B, schistosomiasis, and the common cold. When acquired immunity disappears after some time, the recovered individuals become susceptible again [2]. To incorporate this phenomenon, SIRS epidemic models have emerged.

According to the total population, the SIRS epidemic model can be divided into two kinds: one kind is with constant total population, and the other kind is with varying total population. Theoretically, the analysis on basic properties and global asymptotic stability of SIRS model with varying total population size is more difficult. A large portion of works focus on some special cases of this type of SIRS epidemic model (see [3-5] and the references therein). Mena-Lorca and Hethcote [2] discussed an SIRS epidemic model with simple mass action incidence and constant immigration. They proposed the following open question: when the basic reproduction number is greater than one, the unique endemic equilibrium is globally asymptotically stable. $\mathrm{Ma}$ et al. also proposed the same open question in [6].

Nowadays, these open questions were gradually being solved. Li et al. [7] tried to study the global dynamical behaviors of SIRS model with incidence rate $\beta(N) S I$ and constant immigration. They did obtain the global asymptotic stability of endemic equilibrium; however, this conclusion is valid only in the cases satisfying certain conditions but is not generally true. It is worth celebrating that the global asymptotic stabilities for classical SIRS model with general incidence rate are completely derived in $[3,8]$. The method they used is LaSalle's invariance principle and Lyapunov direct method. This is the most common method to study the local and global asymptotic stability of epidemic models. By using this method, Ma et al. [9] studied the local and global asymptotic stability of a SIQR model. Cui et al. [10] investigated the transmission dynamics of an epidemic model with vaccination, treatment, and isolation. Therefore, we wonder whether the global stability of SIRS model with 
general incidence rate and constant immigration can be obtained through this method or not.

Motivated by these studies, in this paper, we considered an SIRS epidemic model with constant immigration, especially with immigration of infected individuals. Notice that transmission of infection occurs after adequate contacts between the susceptible and the infectious. To better simulate this phenomenon, we considered a general incidence rate $g(I) S$. Like in [8], function $g(I)$ is assumed as a real locally Lipschitz function on $[0,+\infty)$ and satisfied:

(i) $g(0)=0$ and $g(I)>0$ for $I>0$

(ii) $\lim _{I \longrightarrow 0} g(I) / I=\beta>0$, and $g(I) / I$ is continuous and monotonously nonincreasing for $I>0$

The same hypotheses on function $g(I)$ can be found in [11]. This type of incidence rate was first proposed by Capasso and Serio [12] to model the cholera epidemic spread in Bari in 1973. Since then, many authors studied the different special case of $g(I)$; for more details, one can see literature $[11,13-15]$.

Due to the fact that the infectious individuals or the recovery individuals may move from one place to another, in this paper, we assume that the rate constant of input to the total population is $A$, of which fractions $a, b$, and $c$ $(a+b+c=1$ and $a, b, c \geq 0)$ attribute to susceptible, infected, and recovered compartments. That is, the infectious individuals immigrate to infectious class at time $t$ at rate $b A$, and the recovery individuals immigrate to recovery class at time $t$ at rate $c A$. The rate constant for nature death is $d$; thus, $1 / d$ is the average lifetime. The disease-related death rate for infected individuals is $\alpha$. After a period, the infected individuals might be declared cured of the disease and enter to the recovered class $R$ at rate $\gamma$; thus, $1 / \gamma$ is the average recovery time. Due to the fact that the recovered individuals can only have temporary immunity, thus, the recovered individuals might lose their immunity and move to the infected class $I$ at rate $\delta$. Therefore, the objective model in this paper can be given as

$$
\left\{\begin{array}{l}
\frac{\mathrm{d} S}{\mathrm{~d} t}=a A-g(I) S+\delta R-\mathrm{d} S, \\
\frac{\mathrm{d} I}{\mathrm{~d} t}=b A+g(I) S-(\gamma+d+\alpha) I, \\
\frac{\mathrm{d} R}{\mathrm{~d} t}=c A+\gamma I-(\delta+d) R .
\end{array}\right.
$$

In this paper, we mainly use LaSalle's invariance principle and Lyapunov direct method to investigate the global dynamics of model (1). The whole paper is organized as follows. In section 2, the existence and local stability of each equilibrium will be stated. The globally asymptotically stable state of equilibria will be given in the next section. Numerical simulations and conclusion will be listed in the last two sections.

\section{Basic Properties}

The total population number $N=S+I+R$ and satisfies

$$
\frac{\mathrm{d} N}{\mathrm{~d} t}=A-\mathrm{d} N-\alpha I \leq A-\mathrm{d} N,
$$

which implies that limsup $t \rightarrow \infty N(t) \leq(A / d)$. Therefore, the following domain

$$
\Gamma=\left\{(S, I, R) \in \mathbb{R}_{+}^{3} \mid S+I+R \leq \frac{A}{d}\right\} .
$$

is the positively invariant set with respect to model (1).

From the locally sign-preserving property of limit, we know that $\lim _{I \longrightarrow 0} g(I) / I=\beta>0$ implies $g(I) / I=\beta$ in the small domain of zero. Then, the assumption $g(I) / I$ is continuous and monotonously nonincreasing for $I>0 \mathrm{im}$ plies that $g(I) / I \leq \beta$ for $I>0$, that is, $g(I) \leq \beta I$ for $I \geq 0$. As the assumption $g(I) / I$ is continuous and monotonously nonincreasing and also implies that $(g(I) / I)^{\prime} \leq 0$, a direct calculation derived that $g^{\prime}(I) \leq(g(I) / I)$. Then, it follows from $g(0)=0$ and $\lim _{I \longrightarrow 0}(g(I) / I)=\beta \quad$ that $g^{\prime}\left(0^{+}\right)=\lim _{I \longrightarrow 0^{+}}((g(I)-g(0)) /(I-0))=\lim _{I \longrightarrow 0^{+}}$ $(g(I) / I)=\beta$. Therefore, from the assumptions (a) and (b) on $g(I)$, we have

$$
\begin{aligned}
g(I) & \leq \beta I, \\
g^{\prime}(I) & \leq g(I) / I, \\
\beta & =g^{\prime}\left(0^{+}\right) .
\end{aligned}
$$

To obtain the expressions of equilibria, we shall consider model (1) in two cases: one is $b=0$ and the other is $b>0$, which, respectively, imply model (1) without input of infectious individuals and with constant input of infectious individuals.

Case 1. $b=0$.

Any equilibrium of model (1) must satisfy

$$
\left\{\begin{array}{l}
a A-g(I) S+\delta R-\mathrm{d} S=0 \\
g(I) S-(\gamma+d+\alpha) I=0 \\
c A+\gamma I-(\delta+d) R=0 .
\end{array}\right.
$$

One can easily derive that model (1) always has a disease-free equilibrium $E^{0}=(A(\delta+a d) / d(\delta+d), 0$, $c A / \delta+d)$. From van den Driessche and Watmough [16], we can derive that the basic reproduction number is

$$
\mathfrak{R}_{0}=\frac{\beta A(\delta+a d)}{d(\delta+d)(\gamma+d+\alpha)} .
$$

Biologically, the basic reproduction number $\mathfrak{R}_{0}$ presents the average number of secondary infectious produced by single infective individuals, which are introduced into an entirely susceptible population. 
Except disease-free equilibrium (i.e., the solution of (5) with $I=0$ ), equations (5) may have one positive solution, which can be denoted by $E^{*}=\left(S^{*}, I^{*}, R^{*}\right)$. Then, a simple calculation derives the following:

$$
\begin{aligned}
& S^{*}=\frac{(\gamma+d+\alpha) I^{*}}{g\left(I^{*}\right)}, \\
& R^{*}=\frac{c A+\gamma I^{*}}{\delta+d} .
\end{aligned}
$$

And $I^{*}$ is the nonzero solution of the function $\Phi$

$$
\Phi(I)=d(\gamma+d+\alpha) \frac{I}{g(I)}+\left(d+\alpha+\frac{\mathrm{d} \gamma}{\delta+d}\right) I-\frac{A(\delta+a d)}{\delta+d} .
$$

It follows from assumption (ii) that

$$
\begin{aligned}
\Phi^{\prime}(I) & =d(\gamma+\alpha+d)\left(\frac{I}{g(I)}\right)^{\prime}+\left(d+\alpha+\frac{d \gamma}{d+\delta}\right) \\
> & 0, \text { and if }, \\
\lim _{I \longrightarrow 0^{+}} \Phi(I) & =\frac{d(\gamma+\alpha+d)}{\beta}-\frac{A(\delta+a d)}{\delta+d} \\
& =\frac{d(\gamma+\alpha+d)}{\beta}\left(1-\mathfrak{R}_{0}\right)<0 .
\end{aligned}
$$

Moreover,

$$
\begin{aligned}
\Phi\left(\frac{A}{d}\right)= & d(\gamma+d+\alpha) \frac{A / d}{g(A / d)}+\left(d+\alpha+\frac{\mathrm{d} \gamma}{\delta+d}\right) \frac{A}{d} \\
& -\frac{A(\delta+a d)}{\delta+d} \geq d(\gamma+d+\alpha) \frac{A / d}{g(A / d)} \\
& +\left(\alpha+\frac{\mathrm{d} \gamma}{\delta+d}\right) \frac{A}{d}>0 .
\end{aligned}
$$

Therefore, $\Phi(I)$ has a unique positive root in $(0, A / d)$ if $\mathfrak{R}_{0}>1$.

In sum, we have the following result.

Theorem 1. When $b=0$, we have the following:

(i) Model (1) always has a disease-free equilibrium $E^{0}$

(ii) If $\mathfrak{R}_{0}>1$, besides $E_{0}$, model (1) also has a unique endemic equilibrium $E^{*}\left(S^{*}, I^{*}, R^{*}\right)$, where $S^{*}$ and $R^{*}$ are given in (7) and the expression of $I^{*}$ is the unique positive root of $\Phi(I)$ given in (8)

By analyzing the Jacobian matrix, the local stability of equilibria can be given as follows.
Theorem 2. For model (1) with $b=0$, the disease-free equilibrium $E^{0}$ is locally asymptotically stable when $\mathfrak{R}_{0}<1$, and it is unstable when $\mathfrak{R}_{0}>1$, while the unique endemic equilibrium $E^{*}\left(S^{*}, I^{*}, R^{*}\right)$ is locally asymptotically stable when $\mathfrak{R}_{0}>1$.

Proof. Based on $g(0)=0$ and $g^{\prime}\left(0^{+}\right)=\beta$, the Jacobian matrix at equilibrium $E^{0}$ of model (1) can be given as

$$
J\left(E^{0}\right)=\left(\begin{array}{ccc}
-d & -\frac{\beta A(\delta+a d)}{d(\delta+d)} & \delta \\
0 & \frac{\beta A(\delta+a d)}{d(\delta+d)}-(\gamma+d+\alpha) & 0 \\
0 & \gamma & -(\delta+d)
\end{array}\right) .
$$

One can easily obtain that the eigenvalues of the matrix $J\left(E^{0}\right)$ are all less than zero when $\mathfrak{R}_{0}<1$. This implies that $E^{0}$ is locally asymptotically stable. If $\mathfrak{R}_{0}>1$, the matrix $J\left(E^{0}\right)$ has a positive eigenvalue, which implies that $E^{0}$ is unstable.

For the endemic equilibrium $E^{*}$, the Jacobian matrix is given by

$$
J\left(E^{*}\right)=\left(\begin{array}{ccc}
-g\left(I^{*}\right)-d & -S^{*} g^{\prime}\left(I^{*}\right) & \delta \\
g\left(I^{*}\right) & a_{22} & 0 \\
0 & \gamma & -(\delta+d)
\end{array}\right),
$$

where $a_{22}=S^{*} g^{\prime}\left(I^{*}\right)-(\gamma+d+\alpha)$. The characteristic equation of $J\left(E^{*}\right)$ is

$$
\lambda^{3}+a_{1} \lambda^{2}+a_{2} \lambda+a_{3}=0
$$

where

$$
\begin{aligned}
& a_{1}=g\left(I^{*}\right)+\delta+2 d-a_{22}, \\
& a_{2}=(\gamma+\alpha+d) g\left(I^{*}\right)+(\gamma+d)\left(g\left(I^{*}\right)+d\right)-(\delta+2 d) a_{22}, \\
& a_{3}=(\delta+d)(\gamma+\alpha+d) g\left(I^{*}\right)-\delta \gamma g\left(I^{*}\right)-(\delta+d) d a_{22} .
\end{aligned}
$$

It follows from the expression of $S^{*}$ and the conditions (4) of $g(I)$ that $a_{22} \leq 0$ if $\Re_{0}>1$. Hence, $a_{i}>0, i=1,2,3$ and

$$
\begin{aligned}
a_{1} a_{2}-a_{3}= & \left(g\left(I^{*}\right)-a_{22}\right) a_{2} \\
& +(\delta+2 d) a_{2}-a_{3}>\left(g\left(I^{*}\right)-a_{22}\right) a_{2}>0 .
\end{aligned}
$$

By Routh-Hurwitz criterion, we obtain that the endemic equilibrium $E^{*}$ is locally asymptotically stable if $\mathfrak{R}_{0}>1$.

Case 2. $b>0$.

In this case, the existence and local stability of equilibrium for model (1) are stated as follows.

Theorem 3. When $b>0$, model (1) has only one equilibrium, which is the endemic equilibrium $\widehat{E}=(\widehat{S}, \widehat{I}, \widehat{R})$, where 


$$
\begin{aligned}
& \widehat{S}=\widehat{N}-\widehat{I}-\widehat{R}, \\
& \widehat{I}=\frac{A-\mathrm{d} \widehat{N}}{\alpha}, \\
& \widehat{R}=\frac{(c \alpha+\gamma) A-\gamma \mathrm{d} \widehat{N}}{\alpha(\delta+d)} \text { and } \widehat{N} \text { is the unique solution of, } \\
& \frac{g(\widehat{I})}{\widehat{I}}[((\delta+d)(\alpha+d)+\gamma d) N-(\delta+c \alpha+\gamma+d) A] \\
& =\alpha(\delta+d)\left(\gamma+d+\alpha-\frac{b \alpha A}{A-\mathrm{d} N}\right) .
\end{aligned}
$$

in the domain $(0, A / d)$.

Proof. Due to $N=S+I+R$, any equilibrium of model (1) can be given as

$$
\left\{\begin{array}{l}
b A+g(I)(N-I-R)-(\gamma+d+\alpha) I=0 \\
c A+\gamma I-(\delta+d) R=0 \\
A-\mathrm{d} N-\alpha I=0 .
\end{array}\right.
$$

One can easily verify that all the solutions of equation (17) can not contain $I=0$, which means that model (1) does not have disease-free equilibrium. Denote one solution of (17) by $(I, R, N)=(\widehat{I}, \widehat{R}, \widehat{N})$. Then, one can derive that

$$
\begin{aligned}
& \widehat{I}=\frac{A-\mathrm{d} \widehat{N}}{\alpha}, \\
& \widehat{R}=\frac{(c \alpha+\gamma) A-\gamma \mathrm{d} \widehat{N}}{\alpha(d+\delta)},
\end{aligned}
$$

Substituting (18) into the first equation of (17), we have that $\widehat{N}$ is the solution of

$$
\begin{aligned}
& \frac{g(\widehat{I})}{\widehat{I}}[((\delta+d)(\alpha+d)+\gamma d) N-(\delta+c \alpha+\gamma+d) A] \\
& =\alpha(\delta+d)\left(\gamma+d+\alpha-\frac{b \alpha A}{A-\mathrm{d} N}\right),
\end{aligned}
$$

Next, we prove that equation (19) has only one solution in domain $(0,(A / d))$. Denote

$$
\begin{aligned}
& \phi(N)=\frac{g(\widehat{I})}{\widehat{I}}[((\delta+d)(\alpha+d)+\gamma d) N-(\delta+d+c \alpha+\gamma) A] \\
& \psi(N)=\alpha(\delta+d)\left(\gamma+d+\alpha-\frac{b \alpha A}{A-\mathrm{d} N}\right) .
\end{aligned}
$$

Notice that $g(I) / I$ is continuous and monotone nonincreasing for $I>0$, that is, $d / \mathrm{d} I(g(I) / I) \leq 0$. It follows from the expression of $\widehat{I}$ in (18) that

$$
\frac{d}{\mathrm{~d} N}\left(\frac{g(I)}{I}\right)=\frac{d}{\mathrm{~d} I}\left(\frac{g(I)}{I}\right) \frac{\mathrm{d} I}{\mathrm{~d} N}=-\frac{d}{\alpha} \frac{d}{\mathrm{~d} I}\left(\frac{g(I)}{I}\right) \geq 0 .
$$

Therefore, $\phi^{\prime}(N) \geq 0$, which means that $\phi(N)$ is a nondecreasing function of $N$ in $(0, A / d)$. Furthermore, it follows from the expression of $I$ and $\lim _{I \longrightarrow 0^{+}} g(I) / I=\beta>0$ that

$$
\begin{aligned}
\lim _{N \longrightarrow A^{-} / d} \phi(N) & =\lim _{I \longrightarrow 0^{+}} \frac{g(I)}{I}[\alpha(A / d)((1-c) d+\delta)] \\
& =\alpha \beta(A / d)[(1-c) d+\delta]>0, \\
\lim _{N \longrightarrow 0^{+}} \phi(N) & =-\frac{g(A / d)}{(A / d)}(\delta+d+c \alpha+\gamma) A<0 .
\end{aligned}
$$

Meanwhile, one can verify that $\psi^{\prime}(N)<0$ in $(0, A / d)$, $\lim _{N \longrightarrow 0^{+}} \psi(N)>0$, and $\lim _{N \longrightarrow A / d} \psi(N)=-\infty$. Therefore, there exists only one solution in domain $(0, A / d)$ for equation (19). This completes the proof.

Similar to the proof of Theorem 2, the eigenvalues of Jacobian matrix $J(\widehat{E})$ are all negative, which implies the following theorem.

Theorem 4. When $b>0$, the unique endemic equilibrium $\widehat{E}$ of model (1) is locally asymptotically stable.

\section{Global Dynamics of Model (1)}

For the global dynamical behaviors of model (1), we study them in two cases: $b=0$ and $b>0$, as discussed in section 2 . To obtain the globally asymptotic stability of equilibria, we can rewrite model (1) as the following equivalent model:

$$
\left\{\begin{array}{l}
\frac{\mathrm{d} I}{\mathrm{~d} t}=b A+g(I)(N-I-R)-(\gamma+d+\alpha) I, \\
\frac{\mathrm{d} R}{\mathrm{~d} t}=c A+\gamma I-(\delta+d) R, \\
\frac{\mathrm{d} N}{\mathrm{~d} t}=A-\mathrm{d} N-\alpha I .
\end{array}\right.
$$

The dynamical behaviors of model (1) and model (23) are consistent.

We first discuss the global dynamics of model (1) in the case of $b=0$.

\section{Case 1. $b=0$.}

In this case, by considering Lyapunov function $V=I$ and using LaSalle's invariance principle [17], the global stability of the disease-free equilibrium $E^{0}$ can be derived. We can conclude this with following theorem.

Theorem 5. When $b=0$ and $\mathfrak{R}_{0}<1$, then the disease-free equilibrium $E^{0}$ of model (1) is globally asymptotic stability in $\Gamma$.

For the global stability of endemic equilibrium $E^{*}$, we have the following theorem. 
Theorem 6. When $b=0$ and $\mathfrak{R}_{0}>1$, the endemic equilibrium $E^{*}$ for model (1) is globally asymptotically stable in $\Gamma$.

Proof. Notice that $\left(I^{*}, R^{*}, N^{*}\right)$ is the unique solution of (17) when $\mathfrak{R}_{0}>1$. When $b=0$, it follows from the first equation of (17) that $\left(I^{*}, R^{*}, N^{*}\right)$ satisfies

$$
N^{*}-I^{*}-R^{*}-(\gamma+d+\alpha) \frac{I^{*}}{g\left(I^{*}\right)}=0 .
$$

Then, we can rewrite $\mathrm{d} I / \mathrm{d} t$ in (23) as

$$
\begin{aligned}
\frac{\mathrm{d} I}{\mathrm{~d} t}= & g(I)\left[N-I-R-(\gamma+d+\alpha) \frac{I}{g(I)}\right] \\
= & g(I)\left[N-I-R-(\gamma+d+\alpha) \frac{I}{g(I)}-\left(N^{*}\right.\right. \\
& \left.\left.-I^{*}-R^{*}-(\gamma+d+\alpha) \frac{I^{*}}{g\left(I^{*}\right)}\right)\right] .
\end{aligned}
$$

Hence, the following model

$$
\left\{\begin{array}{l}
\frac{\mathrm{d} I}{\mathrm{~d} t}=g(I)\left[\left(N-N^{*}\right)-\left(I-I^{*}\right)-\left(R-R^{*}\right)\right]-g(I)(\gamma+d+\alpha)\left(\frac{I}{g(I)}-\frac{I^{*}}{g\left(I^{*}\right)}\right), \\
\frac{\mathrm{d} R}{\mathrm{~d} t}=\gamma\left(I-I^{*}\right)-(\delta+d)\left(R-R^{*}\right), \\
\frac{\mathrm{d} N}{\mathrm{~d} t}=-d\left(N-N^{*}\right)-\alpha\left(I-I^{*}\right),
\end{array}\right.
$$

is the equivalent model of (23).

Define a Lyapunov function

$$
V=\frac{1}{2 \alpha}\left(N-N^{*}\right)^{2}+\int_{I^{*}}^{I} \frac{u-I^{*}}{g(u)} \mathrm{d} u+\frac{1}{2 \gamma}\left(R-R^{*}\right)^{2} .
$$

One can testify that $V(I, R, N)>0$ except at $I=I^{*}, R=R^{*}, N=S^{*}+I^{*}+R^{*}$. The derivative of $V$ along solutions of (26) is

$$
\begin{aligned}
\left.\frac{\mathrm{d} V}{\mathrm{~d} t}\right|_{(3.2)=} & \frac{1}{2 \alpha}\left(N-N^{*}\right) \frac{\mathrm{d} N}{\mathrm{~d} t}+\frac{I-I^{*}}{g(I)} \frac{\mathrm{d} I}{\mathrm{~d} t}+\frac{1}{2 \gamma}\left(R-R^{*}\right) \frac{\mathrm{d} R}{\mathrm{~d} t} \\
& -\frac{d}{\alpha}\left(N-N^{*}\right)^{2}-\left(I-I^{*}\right)\left(N-N^{*}\right)-\left(I-I^{*}\right)^{2} \\
& +\left(I-I^{*}\right)\left(N-N^{*}\right)-\left(I-I^{*}\right)\left(R-R^{*}\right) \\
& -(\gamma+d+\alpha)\left(I-I^{*}\right)\left(\frac{I}{g(I)}-\frac{I^{*}}{g\left(I^{*}\right)}\right) \\
& +\left(I-I^{*}\right)\left(R-R^{*}\right)-\frac{\delta+d}{\gamma}\left(R-R^{*}\right)^{2} \\
& -\frac{d}{\alpha}\left(N-N^{*}\right)^{2}-\left(I-I^{*}\right)^{2}-\frac{\delta+d}{\gamma}\left(R-R^{*}\right)^{2} \\
& -(\gamma+d+\alpha)\left(I-I^{*}\right)\left(\frac{I}{g(I)}-\frac{I^{*}}{g\left(I^{*}\right)}\right) .
\end{aligned}
$$

The assumption that $g(I) / I$ is a continuous and monotonously nonincreasing function for $I \geq 0$, which means that

$$
\left(I-I^{*}\right)\left(\frac{I}{g(I)}-\frac{I^{*}}{g\left(I^{*}\right)}\right) \geq 0 .
$$

Therefore, $\mathrm{d} V / \mathrm{d} t$ is a negative definite in $\Gamma$. Using the Lyapunov stability theorem in [18], we know that $(I, R, N)=$ $\left(I^{*}, R^{*}, N^{*}\right)$ of $(23)$ is globally asymptotically stable. This completes the proof.

Remark 1. If $c=0$, then $a=1$, and model (1) becomes

$$
\left\{\begin{array}{l}
\frac{\mathrm{d} S}{\mathrm{~d} t}=A-g(I) S+\delta R-\mathrm{d} S \\
\frac{\mathrm{d} I}{\mathrm{~d} t}=g(I) S-(\gamma+d+\alpha) I \\
\frac{\mathrm{d} R}{\mathrm{~d} t}=\gamma I-(\delta+d) R
\end{array}\right.
$$

From Theorems 5 and 6, one can directly obtain that disease-free equilibrium and endemic equilibrium for this model are both globally asymptotically stable. In fact, model (30) is also a special case of literature [8].

Case 2. $b>0$

For $g(I)$, except assumptions (i) and (ii), we add one assumption (iii). That is, for this case, function $g(I)$ satisfies.

(i) $g(0)=0$ and $g(I)>0$ for $I>0$

(ii) $\lim _{I \longrightarrow 0} g(I) / I=\beta>0$, where $g(I) / I$ is continuous and monotonously nonincreasing for $I>0$

(iii) $g(I)$ as a nondecreasing function of $I>0$

There are many incidence rates that satisfy assumptions (i)-(iii), such as bilinear incidence $g(I) S=\beta I S$ stated in [1], 
saturated incidence rate $g(I) S=(\beta I S / 1)+\alpha I$ discussed in [12].

Similar to the proof of the case $b=0$, the following theorem holds.
Theorem 7. When $b>0$, the endemic equilibrium $\widehat{E}$ of model (1) is globally asymptotically stable in $\Gamma$.

Proof. Similarly, we can rewrite model (23) as the following model:

$$
\left\{\begin{array}{l}
\frac{\mathrm{d} I}{\mathrm{~d} t}=g(I)[(N-\widehat{N})-(I-\widehat{I})-(R-\widehat{R})]-g(I)\left[(\gamma+d+\alpha)\left(\frac{I}{g(I)}-\frac{\widehat{I}}{g(\widehat{I})}\right)+b A\left(\frac{1}{g(I)}-\frac{1}{g(\widehat{I})}\right)\right], \\
\frac{\mathrm{d} R}{\mathrm{~d} t}=\gamma(I-\widehat{I})-(\delta+d)(R-\widehat{R}), \\
\frac{\mathrm{d} N}{\mathrm{~d} t}=-d(N-\widehat{N})-\alpha(I-\widehat{I}) .
\end{array}\right.
$$

Define the Lyapunov function by

$$
W=\frac{1}{2 \alpha}(N-\widehat{N})^{2}+\int_{\widehat{I}}^{I} \frac{u-\widehat{I}}{g(u)} \mathrm{d} u+\frac{1}{2 \gamma}(R-\widehat{R})^{2} .
$$

which is the same as given in the case of $b=0$.

Taking the derivative along (31) derives that

$$
\left.\frac{\mathrm{d} W}{\mathrm{~d} t}\right|_{(3.5)}=-\frac{d}{\alpha}(N-\widehat{N})^{2}-(I-\widehat{I})^{2}-\frac{\delta+d}{\gamma}(R-\widehat{R})^{2}-(\gamma+d+\alpha)(I-\widehat{I})\left(\frac{I}{g(I)}-\frac{\widehat{I}}{g(\widehat{I})}\right)+b A(I-\widehat{I})\left(\frac{1}{g(I)}-\frac{1}{g(\widehat{I})}\right) .
$$

The assumption $g(I)$ as a nondecreasing function of $I>0$ implies that $(I-\widehat{I})(1 / g(I)-1 / g(\widehat{I})) \leq 0$. Therefore, $\mathrm{d} W /\left.\mathrm{d} t\right|_{(3.5)}$ is a negative definite. By the Lyapunov stability theorem [18], the globally asymptotic stability of endemic equilibrium is proved.

\section{Numerical Example}

Theorems 5 and 6 show that when $b=0$, the global dynamical behavior of SIRS models completely relies on the basic reproduction number $\mathfrak{R}_{0}$. Theorems 4 and 7 show that when $b>0$, the SIRS model has only one endemic model, which is globally asymptotically stable. In this section, some numerical simulations are run to demonstrate our theoretical results. To do this, we choose $g(I)=\beta I$ and fix the parameters at $A=1, \alpha=0.032, \gamma=0.1, \delta=0.009$, and $d=0.026$.

Case 1. $a=1, b=c=0$. If $\beta=0.0037$, we have $\mathfrak{R}_{0}=$ $0.9<1$ and $E_{0}=(38.46,0,0)$. Then, it follows from Figure 1(a) that the numerical solutions of model (1) converge to $E_{0}$, which is consistent with Theorem 1. Furthermore, we choose $\beta=0.005$. It follows that $\Re_{0}=1.22>1$ and the unique endemic equilibrium $E^{*}=(31.6,1.356,3.85)$. Figure $1(\mathrm{~b})$ shows that the numerical solutions of model (1) converge to $E^{*}$, which is also consistent with Theorem 5 .
Case 2. $a=0.8, b=0$, and $c=0.2$. When taking $\beta=0.0046, \quad$ then $\mathfrak{R}_{0}=0.95<1 \quad$ and $E_{0}=(34.35,0,5.71)$. It follows from Figure 2(a) that all of the numerical solutions of model (1) converge to $E_{0}$, which is consistent with Theorem 6. By choosing $\beta=0.006$, then $\mathfrak{R}_{0}=1.24>1$ and the unique endemic equilibrium $E^{*}=(26.33,1.26,9.32)$. It follows from Figure 2(b) that all the numerical solutions converge to $E^{*}$, which is also consistent with Theorem 6 .

Case 3. $a=0.6, b=0.1$, and $c=0.3$. In this case, model (1) only owns the endemic equilibrium. If we choose $\beta=0.001$, Figure 3 shows that all solutions of model (1) converge to the unique equilibrium $E^{*}=(26.03,0.76,10.74)$, while if we choose $\beta=0.005$, all the solutions of model (1) converge to $E^{*}=(20.62,1.82,13.78)$, which is consistent with Theorem 7 .

Case 4. The constant immigration is $a=0.8, b=0, c=$ 0.2 for the first 100 days, and then it becomes $a=0.8, b=0, c=0.2$. For this case, we choose $\beta=0.0046$, and Figure 4(a) is one scenario of Case 2 when $\mathfrak{R}_{0}=0.95$. In this case, the disease dies out before 100 days. However, if there is an imported disease case at 100 days, this disease will become outbreak again and become an endemic disease if no control measures are 


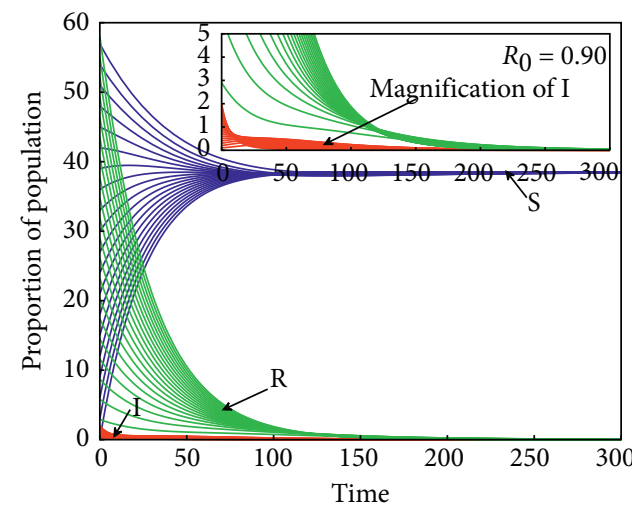

(a)

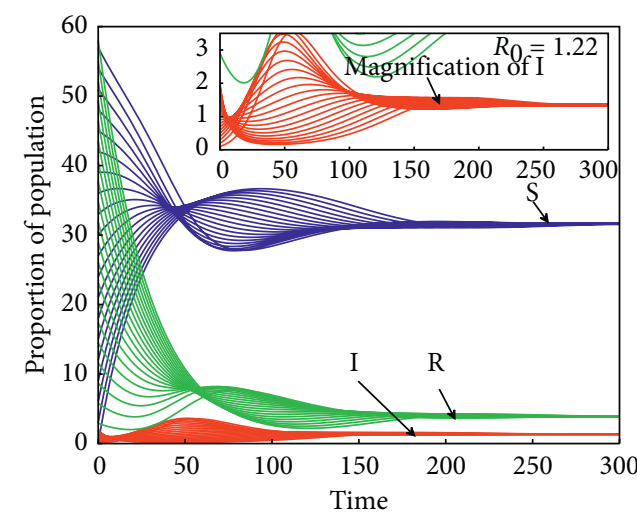

(b)

FIGURE 1: Time plots of populations for the case $a=1, b=0$, and $c=0$ with different initial conditions. (a) $\beta=0.0037$. (b) $\beta=0.005$.

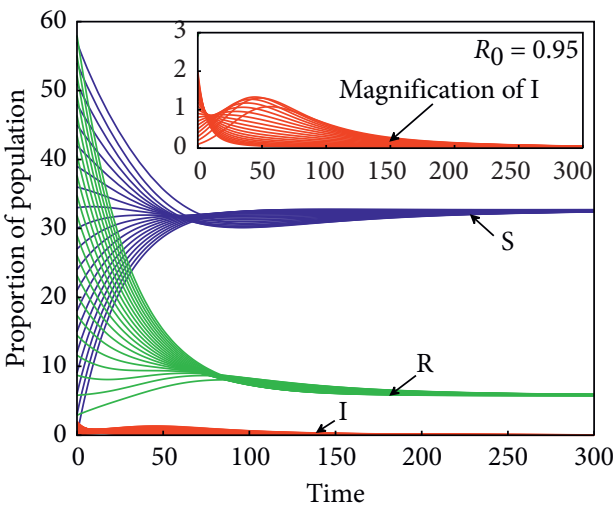

(a)

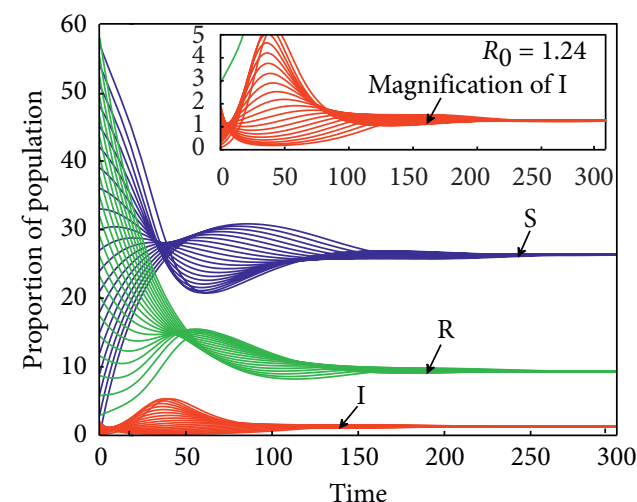

(b)

Figure 2: Time plots of populations for the case $a=0.8, b=0, c=0.2$ with different initial conditions. (a) $\beta=0.0046$. (b) $\beta=0.006$.

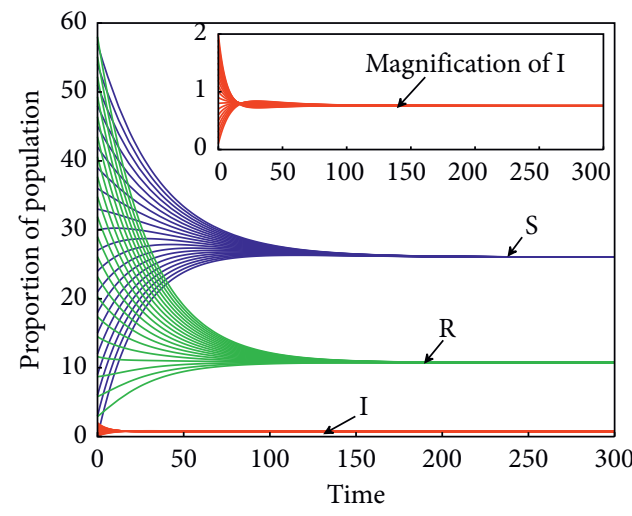

(a)

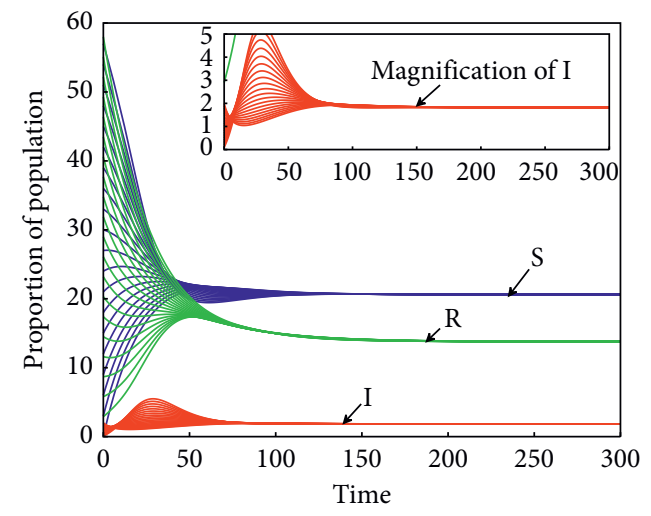

(b)

FIgURE 3: Time plots of populations for the case $a=0.6, b=0.1, c=0.3$ with different initial conditions. (a) $\beta=0.001$. (b) $\beta=0.005$. 


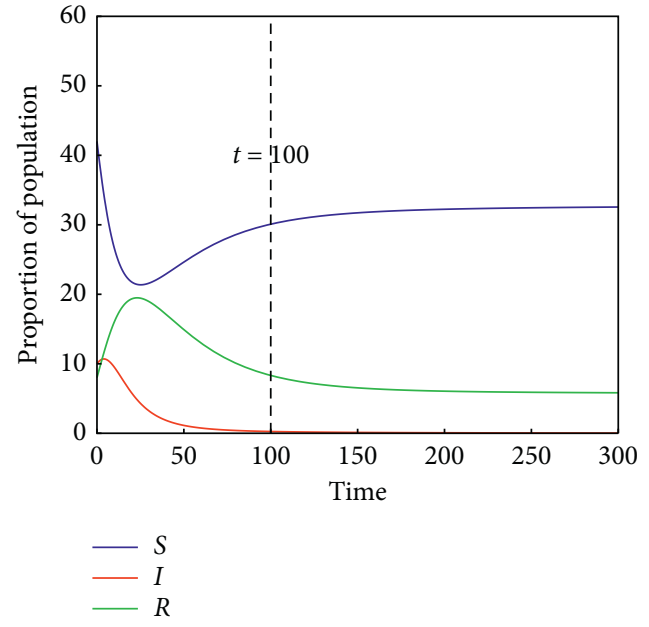

(a)

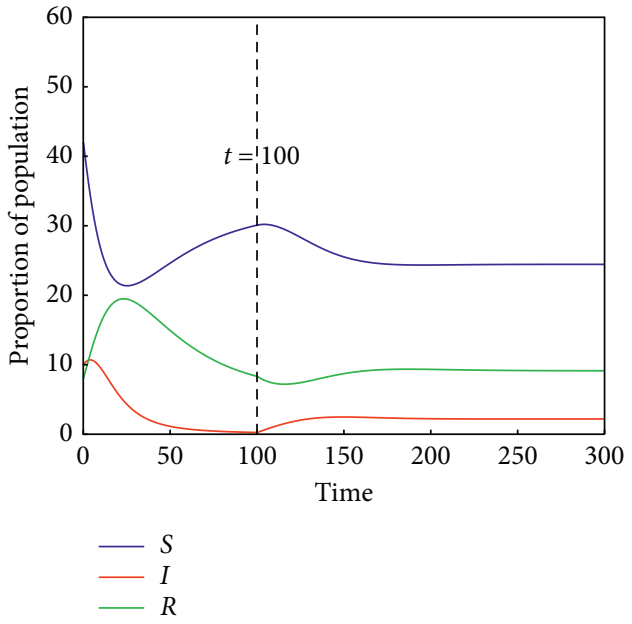

(b)

Figure 4: Time plots of populations for the case $\beta=0.0046$ for different constant immigration. (a) $a=0.8, b=0, c=0.2$. (b) $a=0.8, b=0.1, c=0.1$.

taken. As the results showed in our former work $[19,20]$, the 2019 novel coronavirus (COVID19) will quickly become outbreak in all China in some sense due to the fact that the exposed or infected individuals move from Wuhan (the source of COVID-19) to their hometown during the Spring Festival.

\section{Conclusion}

In this paper, we considered the global properties of SIRS epidemic models with general incidence rate and constant immigration. The results show that when $b=0$, the stability of the model entirely depends on the reproduction number $\mathfrak{R}_{0}$. We should point out that our conclusion is a general result, and it holds for different SIRS models once its incidence rate satisfies our assumptions. In fact, many incidence rates satisfy these assumptions, such as nonmonotone incidence rate with psychological effect $g(I) S=\beta I S / 1+\alpha I^{2}$ and $g(I) S=\beta I S / 1+k I+\alpha I^{2}$, respectively, investigated in $[21,22]$, which satisfy assumptions (i)-(ii), and the bilinear incidence $g(I) S=\beta I S$ stated in [1], the Hattaf-Yousfi functional response $\beta I S / \alpha_{0}+\alpha_{1} S+\alpha_{2} I+\alpha_{3} I S$ [23] with $\alpha_{0}=1, \alpha_{1}=\alpha_{3}=0$ discussed in [12], which satisfy assumptions (i)-(iii).

Biologically, the global asymptotic stability of diseasefree equilibrium means that the disease will die out in the whole population, while the globally asymptotic stability of endemic equilibrium means that the disease will persist in the whole population. Therefore, Theorem 6 implies that the disease will extinct if $\mathfrak{R}_{0}<1$ and Theorem 6 reveals that the disease will persist if $\mathfrak{R}_{0}>1$. When $b>0$, the infection cannot be eliminated due to the constant input of new infections. In this case, model (1) always has only one equilibrium $\widehat{E}$. Theorem 7 shows that the unique endemic equilibrium $\widehat{E}$ is globally asymptotically stable, which means that the disease always exists if the input of infectious individuals size is not zero. Therefore, to control the transmit of infectious disease, we should first shrink the input of infected individuals to zero and then keep the basic reproduction number less than one. For example, COVID-19 has become a widespread outbreak in China, which in part is due to the massive population shift in China during the Spring Festival. Fortunately, the daily new confirmed COVID-19 cases are declining, and we are confident that the disease will soon become extinct under the Chinese government and people's efforts.

\section{Data Availability}

The datasets used or analysed during the current study are available from the authors upon reasonable request.

\section{Conflicts of Interest}

The authors declare that there are no conflicts of interest regarding the publication of this paper.

\section{Acknowledgments}

This work was supported in part by the Natural Science Foundation of Ningxia (No. 2020AAC03060), the Natural Science Project of Ningxia Higher Institutions (No. NGY2020005), the National Natural Science Foundation of China (No. 12001305), and the Major Innovation Projects for Building First-class Universities in China's Western Region (No. ZKZD2017009).

\section{References}

[1] W. O. Kermack and A. G. McKendrick, "A contribution to the mathematical theory of epidemics," in Proceedings of the Royal Society, vol. 115, pp. 700-721, London, UK, 1927.

[2] J. Mena-Lorca and H. W. Hethcote, "Dynamic models of infectious diseases as regulators of population sizes," Journal of Mathematical Biology, vol. 30, pp. 673-716, 1992. 
[3] Y. Bai and X. Mu, "Global asymptotic stability of a generalized SIRS epidemic model with transfer from infectious to susceptible," The Journal of Applied Analysis and Computation, vol. 8, no. 2, pp. 402-412, 2018.

[4] F. Brauer and P. van den Driessche, "Models for transmission of disease with immigration of infectives," Mathematical Biosciences, vol. 171, no. 2, pp. 143-154, 2001.

[5] Q. Tang, Z. Teng, and X. Abdurahman, "A new Lyapunov function for SIRS epidemic models," Bulletin of the Malaysian Mathematical Sciences Society, vol. 40, no. 1, pp. 237-258, 2017.

[6] Z. Ma, Y. Zhou, W. Wang, and Z. Jin, Mathematical Modelling and Study on Epidemic Dynamic Models, Science Press, Beijing, China, 2002.

[7] J.-q. Li, J. Zhang, and Z.-e. Ma, "Global analysis of some epidemic models with general contact rate and constant immigration," Applied Mathematics and Mechanics (English Edition), vol. 25, no. 4, pp. 396-404, 2004.

[8] T. Li, F. Zhang, H. Liu, and Y. Chen, "Threshold dynamics of an SIRS model with nonlinear incidence rate and transfer from infectious to susceptible," Applied Mathematics Letters, vol. 70, pp. 52-57, 2017.

[9] Y. Ma, J.-B. Liu, and H. Li, "Global dynamics of an SIQR model with vaccination and elimination hybrid strategies," Mathematics, vol. 6, no. 12, p. 328, 2018.

[10] Q. Cui, Q. Zhang, Z. Qiu, and X. Yang, "Transmission dynamics of an epidemic model with vaccination, treatment and isolation," Bulletin of the Malaysian Mathematical Sciences Society, vol. 42, no. 3, pp. 885-896, 2019.

[11] J. Li, Y. Yang, Y. Xiao et al., "A class of Lyapunov functions and the global stability of some epidemic models with nonlinear incidence," The Journal of Applied Analysis and Computation, vol. 6, no. 1, pp. 38-46, 2015.

[12] V. Capasso and G. Serio, "A generalization of the KermackMcKendrick deterministic epidemic model," Mathematical Biosciences, vol. 42, no. 1-2, pp. 43-61, 1978.

[13] S. Henshaw and C. C. McCluskey, "Global stability of a vaccination model with immigration," Electronic Journal of Differential Equations, vol. 92, pp. 1-10, 2015.

[14] C. C. McCluskey, "Global stability for an SEI model of infectious disease with age structure and immigration of infecteds," Mathematical Biosciences and Engineering: MBE, vol. 13, no. 2, pp. 381-400, 2016.

[15] R. P. Sigdel and C. C. McCluskey, "Global stability for an SEI model of infectious disease with immigration," Applied Mathematics and Computation, vol. 243, pp. 684-689, 2014.

[16] P. van den Driessche and J. Watmough, "Reproduction numbers and sub-threshold endemic equilibria for compartmental models of disease transmission," Mathematical Biosciences, vol. 180, no. 1-2, pp. 29-48, 2002.

[17] J. P. LaSalle, The Stability of Dynamical Systems, Society for industrial and applied mahtmatics ohicadephia pennsylvania, Philadelphia, PA, USA, 1976.

[18] F. Verbulst, Nonlinear Differential Equations and Dynamical Systems, Springer, Berlin, Germany, 1996.

[19] Q. Cui, Z. Hu, Y. Li, J. Han, Z. Teng, and J. Qian, “Dynamic variations of the COVID-19 disease at different quarantine strategies in Wuhan and mainland China," Journal of Infection and Public Health, vol. 13, no. 6, pp. 849-855, 2020.

[20] Z. Hu, Q. Cui, J. Han, X. Wang, W. Sha, and Z. Teng, "Evaluation and prediction of the COVID-19 variations at different input population and quarantine strategies, a case study in Guangdong province, China," International Journal of Infectious Diseases, vol. 95, pp. 231-240, 2020.
[21] D. Xiao and S. Ruan, "Global analysis of an epidemic model with nonmonotone incidence rate," Mathematical Biosciences, vol. 208, no. 2, pp. 419-429, 2007.

[22] D. Xiao and Y. Zhou, "Qualitative analysis of an epidemic model," The Canadian Applied Mathematics Quarterly, vol. 14, pp. 469-492, 2006.

[23] K. Hattaf and N. Yousfi, "A class of delayed viral infection models with general incidence rate and adaptive immune response," International Journal of Dynamics and Control, vol. 4, no. 3, pp. 254-265, 2016. 